\title{
Study on the Key Technology of Extracorporeal Shock Wave Lithotripsy
}

\author{
Qingbo Miao, a ${ }^{\text {* }}$, Jiangtao Liu ${ }^{1, b}$, Degang Kong ${ }^{1, c}$, \\ Yongwei Yaun ${ }^{1, \mathrm{~d}}$ and Shanshan $\mathrm{Li}^{1, \mathrm{e}}$ \\ 1Mechanical \& Electronic Engineering College, Agricultural university of Hebei, Baoding 071001 \\ amiaoqingbo520@126.com, bliujiangtao2003@126.com, 'kdg198615@163.com, \\ dyyw0314@126.com, ${ }^{\mathrm{d}}$ ndlishanshan@126.com,
}

Keywords: Medical instruments; Stone; Extracorporeal gravel; External shock wave source; Lifting device

\begin{abstract}
In view of the problem that improper positioning of external lithotripsy, frequent replacement of electrodes, poor discharge stability, focus drift and high cost, it is designed a kind of extracorporeal shock wave lithotripsy with X-ray and laser double - positioning. The ADAMS software was used to simulate the extracorporeal crusher lifting device, it can be seen from the simulation results that when the lifting device can achieve the lift between $0-500 \mathrm{~mm}$, it is beneficial to locate the stones in the body and facilitate the lithotripsy of the stones in the body in this interval, the device can improve the success rate of extracorporeal lithotripsy, reduce the pain of patients with external lithotripsy, speed up the process of in vitro gravel and reduce the treatment of stones in patients with time.
\end{abstract}

\section{Introduction}

The stones are common diseases that it can cause renal colic, urinary tract infection and renal insufficiency, cause great pain to the patient. Urolithiasis is one of the common diseases of the urinary system; it is occupying the first place in urological inpatients. Extracorporeal shock wave lithotripsy is the use of shock wave that produce the energy to shatter the stones of the patient, it is a method that treats urinary system calculus by removing urine in vitro, and extracorporeal shock wave lithotripsy treats stones in vitro, it has the advantage of no intubation, positioning accuracy, no pain, no damage to the organ, trauma, low cost, less complications, no need for anesthesia safe and reliable, it is one of the effective treatment of kidney and ureteral stones, it has more than $90 \%$ of patients with urolithiasis from the pain of surgery ${ }^{[1-2]}$. At present, the extracorporeal shock wave lithotripsy has been in clinical application for 35 years, tens of millions of patients have benefited around the world, the foreign research is earlier on the external lithotripsy, Dormer is from Germany and he began to develop in 1969 , it was first applied in clinical practice in $1980^{[3-4]}$, so that the non-surgical treatment of urolithiasis is possible, and become a milestone in the development of urology.

According to the different working principle, the extracorporeal shock wave lithotripsy can be divided into liquid water tank type, liquid electric dry type, double electric pulse and electromagnetic type. The liquid shock wave generator has the advantages that the technology is mature and the safety may be large. It is especially suitable for the treatment of large stones and kidney antlers. However, the shortcomings are frequent replacement electrodes. In general, treating one patient will replace the electrode. Another point is the electrode tip in the discharge process loss, so that the tip of the distance increases, resulting in focus drift. The electromagnetic shock wave generating source has the advantage of stable point of accumulation, easy to offset, no need to replace the electrode, but the generator price is high and to be replaced. The liquid power compound pulse wave source has the advantages of liquid, shorten the time, about $40 \%$ of the gravel effect. The double pulse source is the real direction of development of extracorporeal shock wave lithotripsy ${ }^{[5-6]}$. 


\section{Overall Structure and Operational Principle}

Overall Structure. The overall structure of the external lithotripsy is shown in Fig. 1. The extracorporeal shock wave lithotripsy is mainly composed of frame, lifting device, treatment bed, $\mathrm{C}$-arm, reflective cup, shock wave generating device, laser generating device, display screen and control cabinet. The lifting device comprises guide columns, left and right lifting frame, connecting shafts, a moving rod, a connecting rod, an electric putter and running wheels, as shown in Fig. 2. The laser generating device includes a laser generator, a laser receiver and a positioning system. The laser generating device is mounted on both sides of the shock wave generating device and the stones in the patient are subjected to a method of generating a shock wave Positioning, to achieve the best therapeutic effect. The guide bar is mounted on the frame, the electric putter is mounted on the frame, the left and right lifting frame is mounted on the guide post, the left and right sides of the lift frame are connected together by two connecting rods to achieve synchronous movement, while the left and right lift frame has two The same tripod, and between two identical tripods supported by the connecting shaft to ensure the safety and reliability of the lift, a treatment bed, a shock wave generating device, a laser generating device and a display screen are installed in the upper part of the lifting device and so on, the treatment bed installed below the reflective cup and console, in the reflective cup and console directly above the installation of the $\mathrm{C}$-arm, shock wave generator and laser generating device, easy to find the body of the stones, the display installed in the rear of the $\mathrm{C}$-arm through the $\mathrm{C}$-arm movement to achieve the specific location of the show stones.

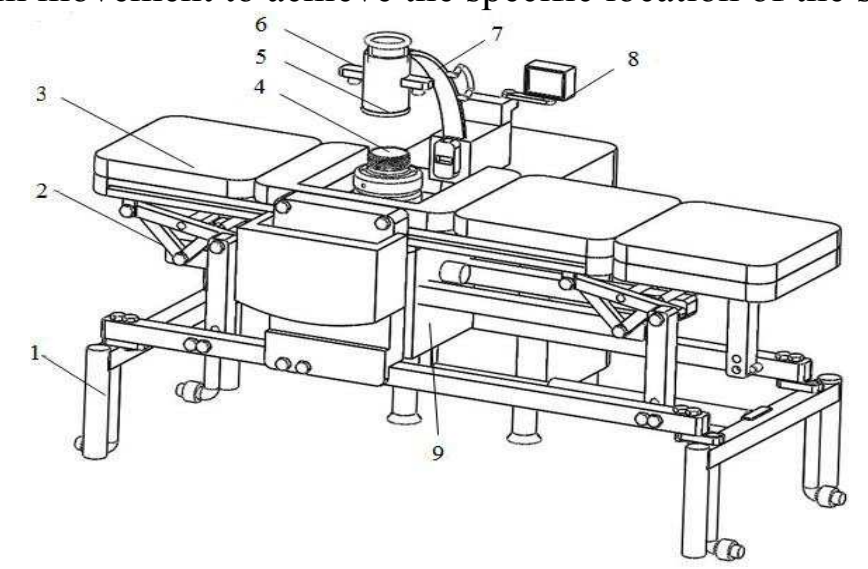

1.frame; 2. lifting device; 3. treatment bed; 4 reflective cup; 5 . shock wave generating device; 6 . laser generating device; 7. C-arm; 8. display screen; 9. control cabinet

Figure 1. The overall structure of extracorporeal shock wave lithotripsy

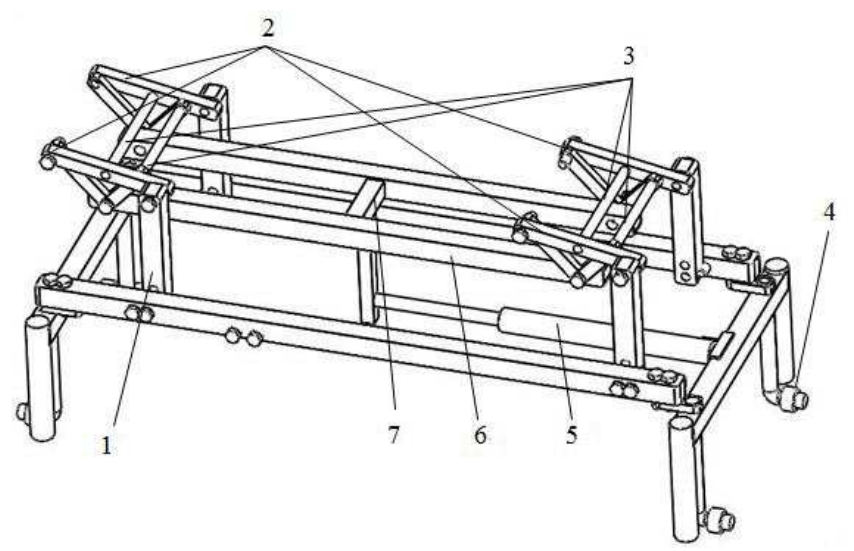

1. guide columns; 2. left and right lifting frame; 3 . connecting shafts; 4. running wheels; 5 . an electric putter; 6 . a moving rod; 7 . a connecting rod

Figure 2. The structure of lifting device 
Operational Principle. Firstly, the patient is lying on the treatment bed, and the electric push rod at the lower part of the treatment bed is driven to adjust the height of the lifting device to meet the comfort of the patient during extracorporeal lithotripsy. The $\mathrm{C}$-arm rotation structure drives the extracorporeal shock wave source above the treatment bed and the laser generator to find the position of the stone in the patient. The position of the stone in the patient is determined by the image on the display screen. It is adopted the X-ray and laser dual positioning method that the $\mathrm{X}$-ray positioning is based on the laser positioning method for the auxiliary, and the position of the stones in the patients can be precisely positioned by the positioning of the X-ray and the laser positioning. The liquid electrical double pulse wave source is used with high pressure in a water discharge, the shock waves can produce a certain amount of electricity in a given time, so that the gas dissolved in the release of water, the shock wave movement produce expansion and collapse double pulse in a very short period of time, the performance of the waveform with liquid pulse makes full use of the cavitation effect. The shock wave is used to generate two shock waves. The first shock wave is gathered around the stone in the patient and a large number of fine cells are generated inside. The time required for the bubble to produce the burst is very short, and when the bubble is generated at the first shock wave, it is resulted in a second shock wave and increased the expansion of the bubble burst. Therefore, the utility model increases the pressure and the pulling force of stones, the gravel effect, shortens the treatment time and reduces the soft tissue scratch.

\section{The Simulation Analysis of Lifting Device}

The Establishment of Simulation Analysis Model for Lifting Device. The three-dimensional software Solidworks is used to draw the parts of the components of the extracorporeal crusher and the components are assembled and imported into the ADAMS dynamic simulation software. The ADAMS simulation model is shown in Fig. 3.

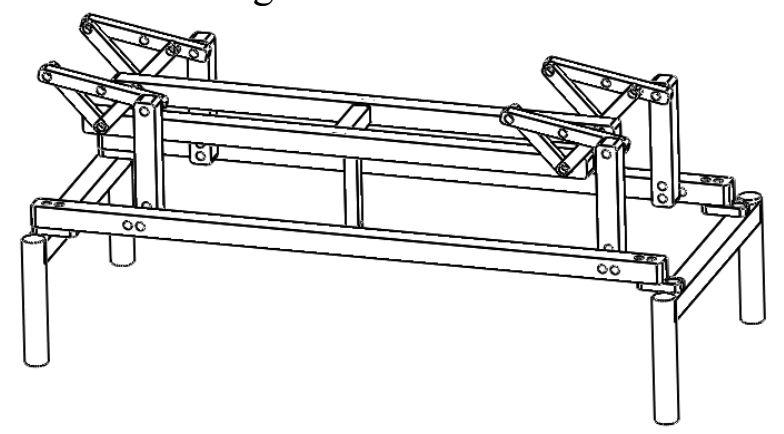

Figure 3. The simulation model of lifting device

First of all, according to the material properties of various parts of the model of the various parts set the material parameters. The guide column, left and right lifting frame and connecting rod material for the stainless steel in Fig. 2, the material properties shown in Table 1. Then, the motion constraint is added to the model, the movement pair is created on the rack and the earth in the model and the vertical movement is simulated. The left and right lifting frame and the guide column, the frame and the guide column are used to create the rotation pair. The next step is to add a force constraint between the two members. The contact force is added to the connecting rod and the moving rod. The contact parameters are set as shown in Table 2 to simulate the constraint relationship between the two. Finally, the whole model is added the motion, according to the actual movement of the lifting device to determine the model's motion function.

Table 1 Material properties

\begin{tabular}{|c|c|c|c|}
\hline material & density $/\left(\mathrm{kg} \cdot \mathrm{m}^{-3}\right)$ & Elastic Modulus $/\left(\mathrm{N} \cdot \mathrm{m}^{-2}\right)$ & Poisson ratio \\
\hline stainless steel & $7.91 \times 10^{3}$ & $7.58 \times 10^{11}$ & 0.3 \\
\hline \multicolumn{4}{|c|}{ Table 2 Material contact parameters } \\
\hline \multicolumn{2}{|c|}{ material } & \multicolumn{2}{|c|}{ Damping coefficient/Mpa } \\
\hline \multicolumn{2}{|c|}{ stainless steel - stainless steel } & \multicolumn{2}{|c|}{10} \\
\hline
\end{tabular}


Results of Simulation Analysis. In order to verify the feasibility of the lifting device and the rationality of the structural design, the lifting device model is established in ADAMS. The displacement curve of the lifting device is obtained by the lifting device model in ADAMS. In the simulation model, a contact constraint is created between the connecting rod and the moving rod, and the contact force is applied to both. In the actual movement, the connecting rod is subjected to the force of the electric push rod, and the moving rod is pushed to move. Simulation of movement, the lifting device at the highest end, lifting device model can move up according to the movement function about $5 \mathrm{~s}$, the lifting device can move down to the lowest point, and then after $5 \mathrm{~s}$, lifting device can rise to the highest point, the entire device takes about 10 s to achieve the lifting process of the device, the lifting curve shown in Fig. 4.

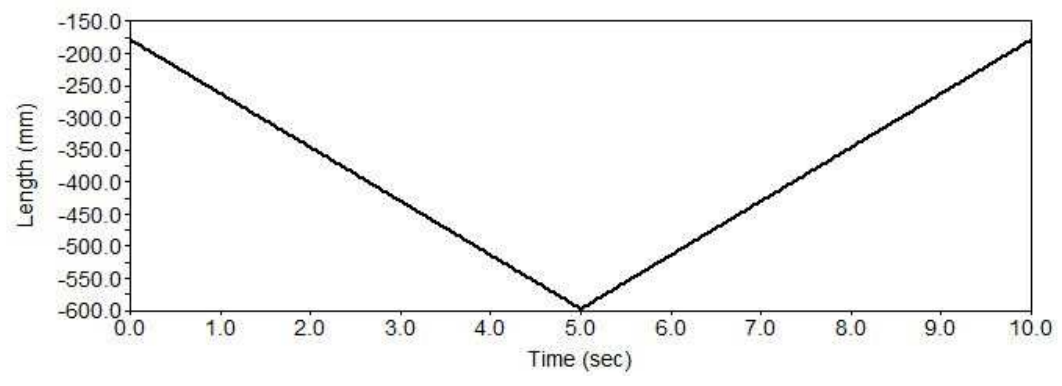

Figure 4. The lift curve

\section{Conclusion}

An extracorporeal shock wave lithotripsy was designed. The structure and principle of extracorporeal shock wave lithotripsy were introduced. The positioning of the stone in the patient was improved by using X-ray positioning and laser positioning method that the X-ray positioning is based on the laser positioning method for the auxiliary, Sex, and the use of liquid-electric pulse-type pulse of the body of the stones in patients with gravel, improve the success rate of gravel. And the use of liquid-electric pulse pulse of the body of the stones in patients with gravel, improve the success rate of gravel. The kinematic analysis of the lifting device is carried out by using ADAMS software, the height of the lifting device has a significant effect on the efficiency of the stones in the treatment of the patient. Taking into account the various factors of in vitro gravel, the lifting device can achieve the lift between $0-500 \mathrm{~mm}$, the stones in the patient can be precisely positioned. And can shorten the time of extracorporeal lithotripsy, so that patients may suffer from less pain with stones, early out of the disease as soon as possible, it is a new idea and method for the study of extracorporeal lithotripsy.

\section{Acknowledgement}

In this paper, the research was sponsored by Science and Technology Research Project of 2016 Baoding (Project No. 16ZG011)

\section{References}

[1] Y.L. Guo: Extracorporeal Shock Wave(Shandong Science and Technology Publishing House, China 2004)

[2] Z.Q. Ye. Guidelines for the Diagnosis and Treatment of Urolithiasis (Beijing: People's Medical Publishing House, China 2009)

[3] C. Chaussy, W. Brendel and E. Schmiedt: Lancet, Vol. 316 (1980) No.8207, p. 1265.

[4] C.G. Chaussy and C.J. Fuchs: The Journal of Urology, Vol. 141 (1989) No.3Part2, p.782.

[5] D.W. Liu and Y.B. Li: Chinese Journal of Medical Device, Vol. 17 (2004) No.1, p.18.

[6] S.H. Ma: Chinese Medical Equipment Journal, Vol. 31 (2010) No.12, p.37. 